\title{
Total synthesis of the novel antifungal agent (士)-Jesterone
}

\author{
Goverdhan Mehta* and Subhas Chandra Pan \\ Department of Organic Chemistry, Indian Institute of Science \\ Bangalore 560 012, India \\ Email Addressgm@orgchem.iisc.ernet.in
}

\section{Supporting Information}<smiles>COc1ccc(O)c(CC=C(C)C)c1</smiles>

\section{Compound 6}

${ }^{1} \mathrm{H}$ NMR $(300 \mathrm{MHz})\left(\mathrm{CDCl}_{3}\right) \delta$ 6.76-6.64 (m, 3H), $5.33(\mathrm{tt}, 1 \mathrm{H}, \mathrm{J}=1.5 \mathrm{~Hz}, 7.5 \mathrm{~Hz}), 5.12$ (br s, 1H), 3.77 (s, 3H), $3.34(\mathrm{~d}, 2 \mathrm{H}, \mathrm{J}=6.9 \mathrm{~Hz}), 1.78(\mathrm{~s}, 6 \mathrm{H})$

${ }^{13} \mathrm{C}$ NMR $(75 \mathrm{MHz})\left(\mathrm{CDCl}_{3}\right) \delta 153.5,148.1,134.5,128.2,121.6,116.1,115.7,111.9$, $55.7,29.7,25.7,17.8$<smiles>CC(C)=CCC1=CC(=O)C=CC1=O</smiles>

\section{Compound 7}

${ }^{1} \mathrm{H}$ NMR $(300 \mathrm{MHz})\left(\mathrm{CDCl}_{3}\right) \delta$ 6.77-6.67 (m, 2H), 6.54-6.52 (m, 1H), 5.17-5.10 (m, $1 \mathrm{H}), 3.11(\mathrm{~d}, 2 \mathrm{H}, \mathrm{J}=6.6 \mathrm{~Hz}), 1.75(\mathrm{~s}, 3 \mathrm{H}), 1.63(\mathrm{~s}, 3 \mathrm{H})$

${ }^{13} \mathrm{C}$ NMR $(75 \mathrm{MHz})\left(\mathrm{CDCl}_{3}\right) \delta 187.9,187.5,148.5,136.7,136.5,136.3,132.3,117.8$, $27.4,25.7,17.7$ 


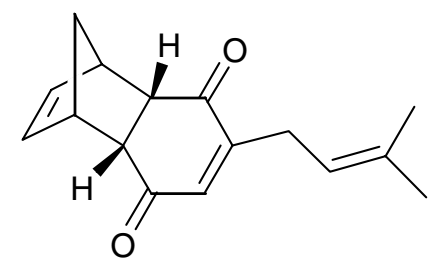

\section{Compound 8}

${ }^{1} \mathrm{H}$ NMR $(300 \mathrm{MHz})\left(\mathrm{CDCl}_{3}\right) \delta 6.39(\mathrm{~s}, 1 \mathrm{H}), 6.05(\mathrm{~d}, 1 \mathrm{H}, \mathrm{J}=2.4 \mathrm{~Hz}), 6.00(\mathrm{~d}, 1 \mathrm{H}, \mathrm{J}=2.4$ $\mathrm{Hz}), 5.07(\mathrm{t}, 1 \mathrm{H}, \mathrm{J}=7.2 \mathrm{~Hz}), 3.51(\mathrm{br} \mathrm{s}, 2 \mathrm{H}), 3.25-3.23(\mathrm{~m}, 2 \mathrm{H}), 2.98(\mathrm{~d}, 2 \mathrm{H}, \mathrm{J}=6.9 \mathrm{~Hz})$, $1.73(\mathrm{~s}, 3 \mathrm{H}), 1.59(\mathrm{~s}, 3 \mathrm{H}), 1.52(\mathrm{~d}, 1 \mathrm{H}, \mathrm{J}=8.7 \mathrm{~Hz}), 1.45(\mathrm{~d}, 1 \mathrm{H}, \mathrm{J}=9 \mathrm{~Hz})$

${ }^{13} \mathrm{C}$ NMR $(75 \mathrm{MHz})\left(\mathrm{CDCl}_{3}\right) \delta 198.8,198.7,153.8,138.0,135.5,135.0,134.5,117.9$, $48.6,48.5,48.4,48.2,27.4,25.3,17.3$

HRMS Calcd for $(\mathrm{M}+\mathrm{K})^{+} \quad: 281.1167$

Found $\quad(\mathrm{M}+\mathrm{K})^{+} \quad: 281.1372$

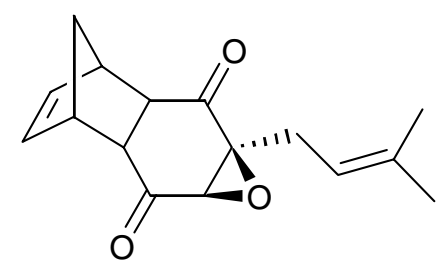

\section{Compound 9}

${ }^{1} \mathrm{H}$ NMR $(300 \mathrm{MHz})\left(\mathrm{CDCl}_{3}\right) \delta 6.05(\mathrm{~s}, 2 \mathrm{H}), 4.92(\mathrm{t}, 1 \mathrm{H}, \mathrm{J}=7.5 \mathrm{~Hz}), 3.50-3.41(\mathrm{~m}, 2 \mathrm{H})$, $3.35(\mathrm{~s}, 1 \mathrm{H}), 3.27$ (br s, 2H), $2.65(\mathrm{dd}, 1 \mathrm{H}, \mathrm{J}=6.9 \mathrm{~Hz}, 15.6 \mathrm{~Hz}), 2.54$ (dd, $1 \mathrm{H} . \mathrm{J}=8.1 \mathrm{~Hz}$, $15.9 \mathrm{~Hz}), 1.68(\mathrm{~s}, 3 \mathrm{H}), 1.59(\mathrm{~s}, 3 \mathrm{H}), 1.48(\mathrm{~d}, 1 \mathrm{H}, \mathrm{J}=8.7 \mathrm{~Hz}), 1.29(\mathrm{~d}, 1 \mathrm{H}, \mathrm{J}=8.4 \mathrm{~Hz})$

${ }^{13} \mathrm{C}$ NMR $(75 \mathrm{MHz})\left(\mathrm{CDCl}_{3}\right) \delta 205.3,205.2,137.0,136.6,115.2,66.9,62.0,50.5,50.1$, $46.7,43.3,43.1,25.8,25.7,18.0$

HRMS Calcd for $(\mathrm{M}+\mathrm{Na})^{+} \quad: 281.1154$

Found $\quad(\mathrm{M}+\mathrm{Na})^{+} \quad: 281.1134$ 


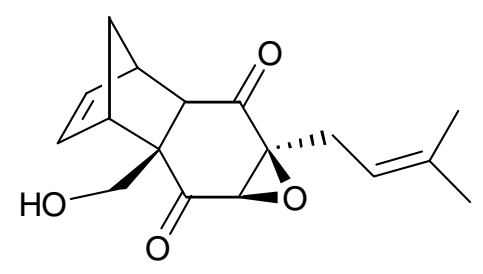

\section{Compound 10}

${ }^{1} \mathrm{H} \mathrm{NMR}(300 \mathrm{MHz})\left(\mathrm{CDCl}_{3}\right) \delta 6.06(\mathrm{br} \mathrm{s}, 2 \mathrm{H}), 4.90(\mathrm{t}, 1 \mathrm{H}, \mathrm{J}=7.5 \mathrm{~Hz}), 4.36(\mathrm{dd}, 1 \mathrm{H}, \mathrm{J}=$ $3.6 \mathrm{~Hz}, 10.8 \mathrm{~Hz}), 3.80(\mathrm{dd}, 1 \mathrm{H}, \mathrm{J}=3.3 \mathrm{~Hz}, 11.4 \mathrm{~Hz}), 3.42(\mathrm{~s}, 1 \mathrm{H}), 3.32-3.28(\mathrm{~m}, 2 \mathrm{H})$, $2.87(\mathrm{~d}, 1 \mathrm{H}, \mathrm{J}=3.6 \mathrm{~Hz}), 2.64-2.55(\mathrm{~m}, 2 \mathrm{H}), 2.17(\mathrm{br} \mathrm{s}, 1 \mathrm{H}), 1.68(\mathrm{~s}, 3 \mathrm{H}), 1.59(\mathrm{~s}, 3 \mathrm{H})$, $1.51(\mathrm{~d}, 1 \mathrm{H}, \mathrm{J}=9.6 \mathrm{~Hz}), 1.44(\mathrm{~d}, 1 \mathrm{H}, \mathrm{J}=9.3 \mathrm{~Hz})$

${ }^{13} \mathrm{C}$ NMR $(75 \mathrm{MHz})\left(\mathrm{CDCl}_{3}\right) \delta$ 205.7, 204.6, 138.1, 137.9, 137.2, 115.0, 68.2, 67.8, 61.8, $61.6,53.9,45.9,44.3,43.4,25.8,25.7,18.0$

HRMS Calcd for $(\mathrm{M}+\mathrm{Na})^{+} \quad: 311.1259$

Found $\quad(\mathrm{M}+\mathrm{Na})^{+} \quad: 311.1250$

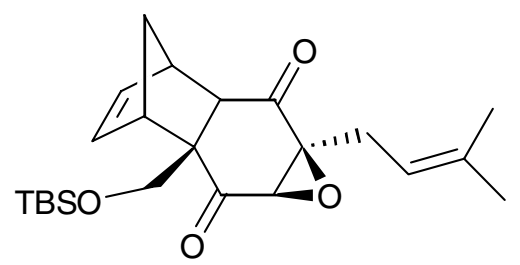

\section{Compound 11}

${ }^{1} \mathrm{H}$ NMR $(300 \mathrm{MHz})\left(\mathrm{CDCl}_{3}\right) \delta$ 6.10-6.01 (m, 2H), 4.94-4.88 (m, 1H), $4.46(\mathrm{~d}, 1 \mathrm{H}, \mathrm{J}=$ $10.5 \mathrm{~Hz}), 3.74(\mathrm{~d}, 1 \mathrm{H}, \mathrm{J}=10.5 \mathrm{~Hz}), 3.38(\mathrm{~s}, 1 \mathrm{H}), 3.27(\mathrm{br} \mathrm{s}, 1 \mathrm{H}), 3.23(\mathrm{br} \mathrm{s}, 1 \mathrm{H}), 2.78(\mathrm{~d}$, $1 \mathrm{H}, \mathrm{J}=3.9 \mathrm{~Hz}), 2.63(\mathrm{dd}, 1 \mathrm{H}, \mathrm{J}=6.6 \mathrm{~Hz}, 15.3 \mathrm{~Hz}), 2.52(\mathrm{dd}, 1 \mathrm{H}, \mathrm{J}=7.8 \mathrm{~Hz}, 15.6 \mathrm{~Hz})$, $1.68(\mathrm{~s}, 3 \mathrm{H}), 1.59(\mathrm{~s}, 3 \mathrm{H}), 1.53(\mathrm{~d}, 1 \mathrm{H}, \mathrm{J}=8.7 \mathrm{~Hz}), 1.39(\mathrm{~d}, 1 \mathrm{H}, \mathrm{J}=9.0 \mathrm{~Hz}), 0.89(\mathrm{~s}, 9 \mathrm{H})$, $0.05(\mathrm{~s}, 6 \mathrm{H})$

${ }^{13} \mathrm{C}$ NMR $(75 \mathrm{MHz})\left(\mathrm{CDCl}_{3}\right) \delta 205.2,204.1,138.7,137.5,136.9,115.2,67.8,62.1$, $61.7,54.1,45.8,43.8,43.2,25.9,25.8,25.7,25.6,18.2,18.0,-5.5,-5.6$

HRMS Calcd for $(\mathrm{M}+\mathrm{Na})^{+} \quad: 425.2124$

Found $\quad(\mathrm{M}+\mathrm{Na})^{+} \quad: 425.2124$ 


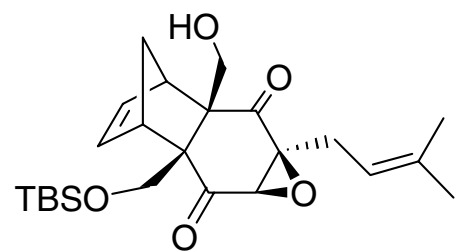

\section{Compound 12}

${ }^{1} \mathrm{H}$ NMR $(300 \mathrm{MHz})\left(\mathrm{CDCl}_{3}\right) \delta 5.96(\mathrm{~s}, 2 \mathrm{H}), 4.82(\mathrm{t}, 1 \mathrm{H}, \mathrm{J}=7.2 \mathrm{~Hz}), 4.08-3.82(\mathrm{~m}, 4 \mathrm{H})$, $3.40(\mathrm{dd}, 1 \mathrm{H}, \mathrm{J}=3.0 \mathrm{~Hz}, 9.6 \mathrm{~Hz}), 3.29(\mathrm{~s}, 1 \mathrm{H}), 3.05(\mathrm{~s}, 1 \mathrm{H}), 2.98(\mathrm{~s}, 1 \mathrm{H}), 2.66(\mathrm{dd}, 1 \mathrm{H}, \mathrm{J}$ $=7.5 \mathrm{~Hz}, 15.3 \mathrm{~Hz}), 2.40(\mathrm{dd}, 1 \mathrm{H}, \mathrm{J}=6.6 \mathrm{~Hz}, 15.3 \mathrm{~Hz}), 1.61(\mathrm{~s}, 3 \mathrm{H}), 1.52(\mathrm{br} \mathrm{s}, 4 \mathrm{H}), 1.23$ $(\mathrm{d}, 1 \mathrm{H}, \mathrm{J}=9.3 \mathrm{~Hz}), 0.83(\mathrm{~s}, 9 \mathrm{H}), 0.01(\mathrm{~s}, 6 \mathrm{H})$

${ }^{13} \mathrm{C}$ NMR $(75 \mathrm{MHz})\left(\mathrm{CDCl}_{3}\right) \delta 206.6,206.3,138.5,138.2,136.6,115.4,68.1,67.6,66.5$, $62.8,62.7,61.8,50.6,50.1,42.7,26.1,25.7,25.6,18.1,17.9,-5.7,-5.8$

HRMS Calcd for $(\mathrm{M}+\mathrm{Na})^{+} \quad: 455.2230$

Found $\quad(\mathrm{M}+\mathrm{Na})^{+} \quad: 455.2205$

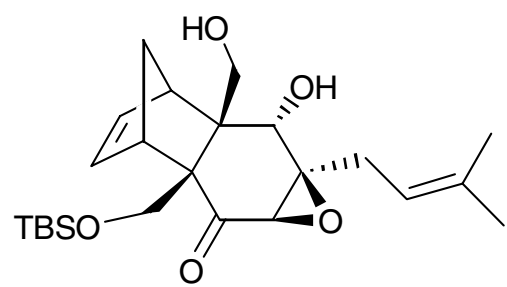

\section{Compound 13}

${ }^{1} \mathrm{H} \mathrm{NMR}(300 \mathrm{MHz})\left(\mathrm{CDCl}_{3}\right) \delta 6.32-6.22(\mathrm{~m}, 2 \mathrm{H}), 5.11(\mathrm{t}, 1 \mathrm{H}, \mathrm{J}=6.9 \mathrm{~Hz}), 4.60(\mathrm{~d}, 1 \mathrm{H}, \mathrm{J}$ $=10.5 \mathrm{~Hz}), 4.21(\mathrm{~d}, 1 \mathrm{H}, \mathrm{J}=9.6 \mathrm{~Hz}), 3.98(\mathrm{dd}, 1 \mathrm{H}, \mathrm{J}=4.8 \mathrm{~Hz}, 11.1 \mathrm{~Hz}), 3.72(\mathrm{~d}, 2 \mathrm{H}, \mathrm{J}=$ $12.9 \mathrm{~Hz}), 3.14(\mathrm{~s}, 1 \mathrm{H}), 3.04(\mathrm{~s}, 1 \mathrm{H}), 2.97(\mathrm{~s}, 1 \mathrm{H}), 2.80(\mathrm{dd}, 1 \mathrm{H}, \mathrm{J}=7.8 \mathrm{~Hz}, 15.0 \mathrm{~Hz}), 2.33$ $(\mathrm{dd}, 1 \mathrm{H}, \mathrm{J}=6.6 \mathrm{~Hz}, 15.0 \mathrm{~Hz}), 2.13(\mathrm{t}, 1 \mathrm{H}, \mathrm{J}=5.1 \mathrm{~Hz}), 1.73(\mathrm{~s}, 3 \mathrm{H}) .1 .63(\mathrm{~s}, 3 \mathrm{H}), 1.30(\mathrm{~d}$, $1 \mathrm{H}, \mathrm{J}=10.2 \mathrm{~Hz}), 1.21(\mathrm{~d}, 1 \mathrm{H}, \mathrm{J}=10.8 \mathrm{~Hz}), 0.87(\mathrm{~s}, 9 \mathrm{H}), 0.03(\mathrm{~s}, 6 \mathrm{H})$

${ }^{13} \mathrm{C}$ NMR $(75 \mathrm{MHz})\left(\mathrm{CDCl}_{3}\right) \delta 207.2,136.9,136.6,116.6,70.9,68.4,65.9,65.4,64.8$, $59.9,56.9,48.1,47.9,44.7,31.0,25.8,25.7,18.1,18.0,-5.5,-5.6$

HRMS Calcd for $(\mathrm{M}+\mathrm{Na})^{+} \quad: 457.2386$

Found $\quad(\mathrm{M}+\mathrm{Na})^{+} \quad: 457.2408$ 
<smiles>CC(C)=CC[C@@H]1O[C@@H]1C(=O)C(CO)=C(CO)C(C)(C)C</smiles>

\section{Compound 14}

${ }^{1} \mathrm{H}$ NMR $(300 \mathrm{MHz})\left(\mathrm{CDCl}_{3}\right) \delta 5.18(\mathrm{t}, 1 \mathrm{H}, \mathrm{J}=7.2 \mathrm{~Hz}), 4.68(\mathrm{~d}, 1 \mathrm{H}, \mathrm{J}=5.7 \mathrm{~Hz}), 4.56$ (dd, $1 \mathrm{H}, \mathrm{J}=4.8 \mathrm{~Hz}, 14.4 \mathrm{~Hz}$ ), 4.47-4.37 (m, 3H), 3.55 (br s, 1H), 3.48 (br s, 1H), 3.34 (s, 1H), $2.91(\mathrm{dd}, 1 \mathrm{H}, \mathrm{J}=7.8 \mathrm{~Hz}, 15.6 \mathrm{~Hz}), 2.43$ (dd, 1H, J = 7.2 Hz, $15.3 \mathrm{~Hz}), 1.73$ (s, 3H), $1.65(\mathrm{~s}, 3 \mathrm{H}), 0.85(\mathrm{~s}, 9 \mathrm{H}), 0.06(\mathrm{~s}, 6 \mathrm{H})$

${ }^{13} \mathrm{C} \mathrm{NMR}(75 \mathrm{MHz})\left(\mathrm{CDCl}_{3}\right) \delta 195.2,154.2,136.7,131.7,116.5,67.8,65.1,62.3,58.2$, $56.1,28.9,25.8,25.7,18.1,18.0,-5.4,-5.5$

HRMS Calcd for $(\mathrm{M}+\mathrm{Na})^{+} \quad: 391.1917$

Found $\quad(\mathrm{M}+\mathrm{Na})^{+} \quad: 391.1935$<smiles>CC(C)=CC[C@@]12O[C@H]1C(=O)C(COS(C)(C)C)=C(C=O)[C@@H]2O</smiles>

\section{Compound 15}

${ }^{1} \mathrm{H}$ NMR $(300 \mathrm{MHz})\left(\mathrm{CDCl}_{3}\right) \delta 10.55(\mathrm{~s}, 1 \mathrm{H}), 5.21$ (br s, $\left.1 \mathrm{H}\right), 5.14(\mathrm{~s}, 1 \mathrm{H}), 4.82(\mathrm{~d}, 1 \mathrm{H}, \mathrm{J}$ $=14.4 \mathrm{~Hz}), 4.65(\mathrm{~d}, 1 \mathrm{H}, \mathrm{J}=14.7 \mathrm{~Hz}), 3.46(\mathrm{~s}, 1 \mathrm{H}), 2.98(\mathrm{br} \mathrm{s}, 1 \mathrm{H}), 2.48$ (br s, 1H), 1.77 (s, 3H), 1.69 (s, 3H), $0.88(\mathrm{~s}, 9 \mathrm{H}), 0.09(\mathrm{~s}, 6 \mathrm{H})$

${ }^{13} \mathrm{C}$ NMR $(75 \mathrm{MHz})\left(\mathrm{CDCl}_{3}\right) \delta 196.4,194.5,143.1,141.9,136.3,115.6,64.2,62.8$, $57.7,56.3,28.3,25.2,25.0,17.4,-6.1,-6.2$

HRMS Calcd for $(\mathrm{M}+\mathrm{Na})^{+} \quad: 389.1760$

Found $\quad(\mathrm{M}+\mathrm{Na})^{+} \quad: 389.1746$ 
<smiles>CC(=O)O[C@H]1C(C=O)=C(COS(C)(=O)=O)C(=O)[C@@H]2O[C@@H](CC=C(C)C)[C@@H]21</smiles>

\section{Compound 16}

${ }^{1} \mathrm{H}$ NMR $(300 \mathrm{MHz})\left(\mathrm{CDCl}_{3}\right) \delta 10.41(\mathrm{~s}, 1 \mathrm{H}), 6.29(\mathrm{~s}, 1 \mathrm{H}), 5.06(\mathrm{t}, 1 \mathrm{H}, \mathrm{J}=7.2 \mathrm{~Hz}), 4.81$ $(\mathrm{d}, 1 \mathrm{H}, \mathrm{J}=14.7 \mathrm{~Hz}), 4.62(\mathrm{~d}, 1 \mathrm{H}, \mathrm{J}=14.7 \mathrm{~Hz}), 3.46(\mathrm{~s}, 1 \mathrm{H}), 2.60(\mathrm{dd}, 1 \mathrm{H}, \mathrm{J}=6.6 \mathrm{~Hz}$, $15.3 \mathrm{~Hz}$ ), 2.49 (dd, 1H, J = 7.8 Hz, $15.6 \mathrm{~Hz}$ ), 2.03 (s, 3H), 1.72 (s, 3H), 1.59 (s, 3H), 0.85 $(\mathrm{s}, 9 \mathrm{H}), 0.06(\mathrm{~s}, 6 \mathrm{H})$

${ }^{13} \mathrm{C}$ NMR $(75 \mathrm{MHz})\left(\mathrm{CDCl}_{3}\right) \delta 197.0,192.1,169.2,144.4,140.2,137.1,115.2,64.7$, $62.6,57.6,57.0,28.7,25.7,25.6,20.6,18.0,17.9,-5.5,-5.6$

HRMS Calcd for $(\mathrm{M}+\mathrm{Na})^{+} \quad: 431.1866$

Found $\quad(\mathrm{M}+\mathrm{Na})^{+} \quad: 431.1866$<smiles></smiles>

\section{Compound 17b}

${ }^{1} \mathrm{H}$ NMR $(300 \mathrm{MHz})\left(\mathrm{CDCl}_{3}\right) \delta 6.65(\mathrm{dd}, 1 \mathrm{H}, \mathrm{J}=1.2 \mathrm{~Hz}, 15.6 \mathrm{~Hz}), 6.41-6.29(\mathrm{~m}, 1 \mathrm{H})$, $6.32(\mathrm{~s}, 1 \mathrm{H}), 5.07(\mathrm{t}, 1 \mathrm{H}, \mathrm{J}=8.1 \mathrm{~Hz}), 4.52(\mathrm{~d}, 1 \mathrm{H}, \mathrm{J}=12 \mathrm{~Hz}), 4.41(\mathrm{~d}, 1 \mathrm{H}, \mathrm{J}=12 \mathrm{~Hz})$, $3.38(\mathrm{~s}, 1 \mathrm{H}), 2.57-2.43(\mathrm{~m}, 2 \mathrm{H}), 2.05(\mathrm{~s}, 3 \mathrm{H}), 1.88(\mathrm{dd}, 3 \mathrm{H}, \mathrm{J}=1.5 \mathrm{~Hz}, 6.6 \mathrm{~Hz}), 1.72(\mathrm{~s}$, $3 \mathrm{H}), 1.59(\mathrm{~s}, 3 \mathrm{H}), 0.85(\mathrm{~s}, 9 \mathrm{H}), 0.04(\mathrm{~s}, 6 \mathrm{H})$

${ }^{13} \mathrm{C}$ NMR $(75 \mathrm{MHz})\left(\mathrm{CDCl}_{3}\right) \delta 195.5,169.9,145.6,136.6,135.6,131.8,127.5,115.8$, $66.1,61.7,57.1,55.8,29.0,25.8,20.9,19.6,18.2,18.0,-5.2,-5.3$

HRMS Calcd for $(\mathrm{M}+\mathrm{Na})^{+} \quad: 443.2230$

Found $\quad(\mathrm{M}+\mathrm{Na})^{+} \quad: 443.2246$ 
<smiles></smiles>

\section{Compound 18}

${ }^{1} \mathrm{H}$ NMR $(300 \mathrm{MHz})\left(\mathrm{CDCl}_{3}\right) \delta 6.69(\mathrm{dd}, 1 \mathrm{H}, \mathrm{J}=1.5 \mathrm{~Hz}, 15.6 \mathrm{~Hz}), 6.53-6.43(\mathrm{~m}, 1 \mathrm{H})$, $5.21(\mathrm{t}, 1 \mathrm{H}, \mathrm{J}=7.5 \mathrm{~Hz}), 4.90(\mathrm{~d}, 1 \mathrm{H}, \mathrm{J}=6 \mathrm{~Hz}), 4.48(\mathrm{~d}, 1 \mathrm{H}, \mathrm{J}=11.7 \mathrm{~Hz}), 4.39$ (d, 1H, $\mathrm{J}=11.7 \mathrm{~Hz}), 3.35(\mathrm{~s}, 1 \mathrm{H}), 2.93(\mathrm{dd}, 1 \mathrm{H}, \mathrm{J}=7.5 \mathrm{~Hz}, 15.6 \mathrm{~Hz}), 2.52(\mathrm{dd}, 1 \mathrm{H}, \mathrm{J}=7.2 \mathrm{~Hz}$, $15 \mathrm{~Hz}), 2.18(\mathrm{~d}, 1 \mathrm{H}, \mathrm{J}=6 \mathrm{~Hz}), 1.95(\mathrm{dd}, 3 \mathrm{H}, \mathrm{J}=1.5 \mathrm{~Hz}, 7.2 \mathrm{~Hz}), 1.76(\mathrm{~s}, 3 \mathrm{H}), 1.68$ (s, $3 \mathrm{H}), 0.86(\mathrm{~s}, 9 \mathrm{H}), 0.06(\mathrm{~s}, 6 \mathrm{H})$

${ }^{13} \mathrm{C}$ NMR $(75 \mathrm{MHz})\left(\mathrm{CDCl}_{3}\right) \delta 195.7,149.2,136.7,135.9,129.6,127.9,116.6,65.7$, $63.8,57.9,55.6,29.1,25.9,25.8,19.5,18.2,18.0,-5.2,-5.3$

HRMS Calcd for $(\mathrm{M}+\mathrm{Na})^{+} \quad: 401.2124$

Found $\quad(\mathrm{M}+\mathrm{Na})^{+} \quad: 401.2135$<smiles></smiles>

\section{Compound 19}

${ }^{1} \mathrm{H}$ NMR $(300 \mathrm{MHz})\left(\mathrm{CDCl}_{3}\right) \delta$ 6.71-6.59 (m, 1H), $6.46(\mathrm{dd}, 1 \mathrm{H}, \mathrm{J}=1.5 \mathrm{~Hz}, 14.7 \mathrm{~Hz})$, $5.01(\mathrm{t}, 1 \mathrm{H}, \mathrm{J}=7.2 \mathrm{~Hz}), 4.59(\mathrm{~d}, 1 \mathrm{H}, \mathrm{J}=11.4 \mathrm{~Hz}), 4.33(\mathrm{~d}, 1 \mathrm{H}, \mathrm{J}=11.4 \mathrm{~Hz}), 3.66(\mathrm{~s}, 1 \mathrm{H})$, $2.85(\mathrm{dd}, 1 \mathrm{H}, \mathrm{J}=7.8 \mathrm{~Hz}, 15.3 \mathrm{~Hz}), 2.66(\mathrm{dd}, 1 \mathrm{H}, \mathrm{J}=6.9 \mathrm{~Hz}, 15.3 \mathrm{~Hz}), 1.93$ (dd, 3H, J = $1.5 \mathrm{~Hz}, 6.6 \mathrm{~Hz}), 1.71(\mathrm{~s}, 3 \mathrm{H}), 1.65(\mathrm{~s}, 3 \mathrm{H}), 0.87(\mathrm{~s}, 9 \mathrm{H}), 0.08(\mathrm{~s}, 3 \mathrm{H}), 0.07(\mathrm{~s}, 3 \mathrm{H})$

${ }^{13} \mathrm{C}$ NMR $(75 \mathrm{MHz})\left(\mathrm{CDCl}_{3}\right) \delta 194.7,193.6,142.5,140.9,137.1,136.6,123.1,115.4$, 61.6, 57.6, 56.4, 25.8, 25.7, 25.6, 20.1, 18.2, 18.0, -5.3, -5.4

HRMS Calcd for $(\mathrm{M}+\mathrm{Na})^{+} \quad: 399.1968$

Found $\quad(\mathrm{M}+\mathrm{Na})^{+} \quad: 399.1973$ 
<smiles>C/C=C/C1=C(CO)C(=O)[C@@H]2O[C@@]2(CC=C(C)C)C1=O</smiles>

\section{Compound 20}

${ }^{1} \mathrm{H}$ NMR $(300 \mathrm{MHz})\left(\mathrm{CDCl}_{3}\right) \delta$ 6.57-6.45 (m, 1H), 6.37-6.30 (m, 1H), 5.04-4.98 (m, 1H), $4.54(\mathrm{~d}, 1 \mathrm{H}, \mathrm{J}=12 \mathrm{~Hz}), 4.43(\mathrm{~d}, 1 \mathrm{H}, \mathrm{J}=12 \mathrm{~Hz}), 3.67(\mathrm{~s}, 1 \mathrm{H}), 2.88(\mathrm{dd}, 1 \mathrm{H}, \mathrm{J}=8.1 \mathrm{~Hz}$, $15.3 \mathrm{~Hz}$ ), 2.64 (dd, 1H, J = 7.2 Hz, 15.6 Hz), 2.41 (br s, 1H), 1.94 (dd, 3H, J = 1.8 Hz, 6.6 $\mathrm{Hz}), 1.72(\mathrm{~s}, 3 \mathrm{H}), 1.65$ (s, 3H)

${ }^{13} \mathrm{C}$ NMR $(75 \mathrm{MHz})\left(\mathrm{CDCl}_{3}\right) \delta 195.3,194.0,142.2,141.8,137.3,136.5,122.1,115.2$, $61.7,57.6,57.4,25.8,25.7,20.0,18.0$

HRMS Calcd for $(\mathrm{M}+\mathrm{Na})^{+} \quad: 285.1103$

Found $\quad(\mathrm{M}+\mathrm{Na})^{+} \quad: 285.1110$<smiles>C/C=C/C1=C(CO)[C@H](O)[C@H]2O[C@@]2(CC=C(C)C)C1=O</smiles>

\section{Compound 1}

${ }^{1} \mathrm{H}$ NMR $(300 \mathrm{MHz})\left(\mathrm{CDCl}_{3}\right) \delta 6.09(\mathrm{~d}, 1 \mathrm{H}, \mathrm{J}=15.9 \mathrm{~Hz}), 5.92-5.81(\mathrm{~m}, 1 \mathrm{H}), 5.06(\mathrm{t}, 1 \mathrm{H}$, $\mathrm{J}=7.2 \mathrm{~Hz}), 4.93(\mathrm{~s}, 1 \mathrm{H}), 4.75(\mathrm{~d}, 1 \mathrm{H}, \mathrm{J}=14.4 \mathrm{~Hz}), 4.48(\mathrm{~d}, 1 \mathrm{H}, \mathrm{J}=14.4 \mathrm{~Hz}), 3.67(\mathrm{~d}$, $1 \mathrm{H}, \mathrm{J}=1.8 \mathrm{~Hz}), 3.20(\mathrm{~d}, 1 \mathrm{H}, \mathrm{J}=3.9 \mathrm{~Hz}), 2.78(\mathrm{dd}, 1 \mathrm{H}, \mathrm{J}=8.1 \mathrm{~Hz}, 15.6 \mathrm{~Hz}), 2.55(\mathrm{dd}$, $1 \mathrm{H}, \mathrm{J}=6.6 \mathrm{~Hz}, 15.6 \mathrm{~Hz}), 2.18(\mathrm{br} \mathrm{s}, 1 \mathrm{H}), 1.84(\mathrm{dd}, 3 \mathrm{H}, \mathrm{J}=1.5 \mathrm{~Hz}, 6.6 \mathrm{~Hz}), 1.71(\mathrm{~s}, 3 \mathrm{H})$, $1.65(\mathrm{~s}, 3 \mathrm{H})$

${ }^{13} \mathrm{C}$ NMR $(75 \mathrm{MHz})\left(\mathrm{CDCl}_{3}\right) \delta 195.0,145.5,136.0,134.9,131.5,122.2,116.5,65.3$, $63.1,60.1,59.5,26.6,25.8,19.2,18.0$

HRMS Calcd for $(\mathrm{M}+\mathrm{Na})^{+} \quad: 287.1259$

Found $\quad(\mathrm{M}+\mathrm{Na})^{+} \quad: 287.1255$ 